\title{
Study of Photon Propagation with various Energies through Bone by Monte Carlo Simulation
}

\author{
A. Yavar ${ }^{\text {a, }}$, I. Forouzankia ${ }^{a}$, S. Mohammadi ${ }^{\mathrm{a}}$, M. F. Rahimi ${ }^{\mathrm{b}}$ \\ ${ }^{a}$ Department of Physics, Faculty of Science, Payame Noor University of Mashhad, Moalem 71 avenue, \\ Mash ad, post code 43391735, Iran \\ ${ }^{b}$ Department of Physics, Faculty of Science, Ferdowsi University, Azadi square, Mashhad, post code \\ 9177948974, Iran
}

\begin{abstract}
The aim of radiation therapy is to maximise the dose to the tumour, while minimising the dose to healthy tissue. The Monte Carlo (MC) simulation by advantages of high accuracy, low cost, and describing the coupled transport of electrons and photons in complex geometries is used increasingly in radiation therapy. Since 50-80\% of the human body is water, many studies have carried out the photons propagation in tissue, although no serious study has performed in bone. The bone, because of its high density, as well high ability of scattering and absorption of photons is important matter in radiation therapy. The aim of present work was the $M C$ simulation of photons propagation with various energies in bone. The MC simulation was written in Fortran 77 program. Emigration of photons in bone was determined using MCNP-4C code to evaluate the results of MC method. The depth of first interaction of photons 1,5 , and $10 \mathrm{MeV}$ in bone were determined in 2.26, 2.90, and $4.40 \mathrm{~cm}$ using the MC method, which were a good consistent with those of MCNP results 2, 3, 4 $\mathrm{cm}$, respectively.
\end{abstract}

Keywords: Monte Carlo simulation, photon propagation, bone, MCNP code

\section{Introduction}

Understanding the propagation and the distribution of light in biological tissue and bone is essential for safe and effective applications of photons in medical diagnostics and therapeutics. The Monte Carlo simulation is, at present, the most accurate method for depicting the coupled transport of electrons and photons in complex geometries, residual inaccuracies may even so exist. The Monte Carlo method can commonly be depicted as a statistical method for numerical integration with the use of random numbers [1-5]. First descriptions of this method reach back to the year 1977 and the first common patterns were computer based computations for radiation transport at the end of World War II. The employ of digital computers made the application of this method a helpful tool in science and technology. Commonly spoken, the Monte Carlo simulation of radiation transport utilizes the knowledge of individual, microscopic particle interactions in matter with the corresponding probability distributions. It uses random numbers to depict the random trajectories of these particles by sampling from the underlying probability distributions. Various sampling techniques utilizing regularly distributed random numbers $(0,1)$ exist. The probability distributions are established on the differential cross sections for the interaction mechanisms [1-7].

For the energies employed in radiotherapy, photons can go through Rayleigh scattering, photoelectric absorption, Compton scattering or production of an electron/positron pair in the electromagnetic field of atoms. The number of photons passing a certain depth of media is reduced exponentially by these interactions. The probability for the occasion of the single processes and the resulting change of energy and direction of produced particles and the incident photon is governed by the differential cross sections. They are contingent upon the atomic number of the medium and the energy of the photon and are established on theoretical or semi-empirical values. The photoelectric effect is the commanding interaction of photons with matter, for those photons whose energy occurs on roughly in the range $1 \mathrm{keV}$ to $0.5 \mathrm{MeV}$. At energy around $1 \mathrm{MeV}$, Compton scattering is typically the most general interaction, because the photoelectric effect and pair production are even less probable. The probability of pair production effect rises hastily as the photon energy raises and becomes the main interaction for energy more than $10 \mathrm{MeV}$ [8-12].

Radiotherapy as a significant type of cancer treatment intends at annihilation of tumour cells with the utilization of ionizing radiation. A consistent quality assurance method is obligatory to certify the accurate dose delivery to a tumour volume and to avoid any needless harm to healthy tissue. The dose, or level of radiation, is recommended by an oncologist, and depends on the size, stage and location of the tumour, and the utilization of any other treatment modalities. The term radiotherapy does not include the utilization of non-ionising radiation to excite radiosensitisers. Tumours cannot be treated in isolation; unavoidably healthy tissue will also be damaged. The purpose of radiation therapy is to maximise the dose to tumour, even as minimising the dose to healthy tissue, that is, to supply the greatest therapeutic ratio.Tracing of photons propagated in tissue and bone 
can be useful to achieve to this purpose. Together with the assist of a computer and MC codes, beam modelling has turn into helpful to supply a reference dose assess for a planned treatment. The validation of these dose calculation algorithms is generally fulfilled by comparisons with measured data. The consistency of measured data sets is however very dependent on several aspects, such as the stability of the accelerator or the choice of detector and experimental set-up. These limitations may thereby limit the number of comparison points and introduce dosimetric problems to the verification [8-12]. Several studies signify that the radiation damage is intolerable if the therapy is utilized before the age of three years, as it can irremediably affect the normal brain growth. Considering that high grade glioma is one of the most forms of pediatric cancer, this represents a very serious restriction to the employ of standard radiotherapy methods: late effects on normal brain may considerably affect the quality of life in long-term survivors [3].The many studies have been carried out on photon propagation in tissue, however, basic studies on photon propagation in bone are scarce, as well interaction cross sections of photon in bone is higher than tissue because of its higher density. Therefore, this study aims to simulate photon propagation in bone with the intention of challenging situations where conventional dose computation algorithms have illustrated some limitations and it is very hard to measure using typical clinical dosimetric procedures. This simulation was included to determine the coordinate of first interaction of photon with various energy in bone, and exiting angle of photon from bone with the aim of determination the widen of photon beam during cross through from bone.

\section{Material And Methods}

Monte Carlo simulation

Monte Carlo simulation was used to trace photon propagation in bone via the Fortran 77 program. Atomic number of bone is 12.31 and the photons 1,5 , and $10 \mathrm{MeV}$ were used in present work, therefore, the most important interactions of photons with bone were Photoelectric effect and Compton scattering.Cross section of Photoelectric effect for per atom defined as [8, 10-11]:

$\sigma_{p h}=4 \alpha^{4} \sqrt{2} Z^{5} \phi_{0}\left(m_{e} c^{2} / E\right)^{7 / 2}$

where $\phi_{0}=8 \pi r_{e}^{2} / 3=6.651 \times 10^{-25} \mathrm{~cm}^{2} ; \alpha=1 / 137 ; c=3 \times 10^{8} \mathrm{~m} / \mathrm{s} ; m_{e}=9.1 \times 10^{-31} \mathrm{~kg} ; \mathrm{Z}$ is atomic number, and $\mathrm{E}$ is energy of photon. As well the cross section of Compton scattering is defined as [10-11]:

$\sigma_{S C}=2 \pi r_{e}^{2}\left\{\frac{1+\gamma}{\gamma^{2}}\left[\frac{2(1+\gamma)}{1+2 \gamma}-\frac{1}{\gamma} \ln (1+2 \gamma)\right]+\frac{1}{2 \gamma} \ln (1+2 \gamma)-\frac{1+3 \gamma}{(1+2 \gamma)^{2}}\right\}$

where $r_{e}$ is electron radius and $\gamma=\frac{E}{m_{e} c^{2}}$. The total cross section of photon is determined as [10-11]:

$\sigma_{t o t}=\sigma_{p h}+Z \sigma_{S C}$

along with $\mathrm{MC}$ simulation, if $\frac{Z \sigma_{s c}}{\sigma_{t o t}}>\delta$, that $\delta$ is random number, therefore photon is scattered and subsequently traversed distance and move direction of photon were calculated by [10-11]:

$\theta=a \cos (2 \delta-1)$

$\phi=2 \pi \delta$

$$
S=\frac{-\operatorname{Ln}(\delta)}{\mu_{t o t}}
$$

where $\theta$ and $\phi$ are scattering angles of photon related to $\mathrm{X}$ and Y axes, respectively (it was assumed direction of initial photon is $Z$ axis). The $\mu_{t o t}$ is total linear attenuation coefficient, $\mu_{t o t}=\frac{\rho N_{A} Z}{A} \sigma_{t o t}$ that $\rho$ is density of matter; $N_{A}$ is Avogadro number and A is atomic mass of matter. The energy of scattered photon can be calculated as [10-11]:

$$
E^{\prime}=\frac{E}{1+\gamma(1-\cos \theta)}
$$

where $E^{\prime}$ is energy of photon after scattering, $E$ is energy of initial photon.

Figure 1 illustrates flowchart of photon interactions with bone $\left(30 \times 30 \times 30 \mathrm{~cm}^{3}\right)$ using MC method.

Once launched, the photon was moved a distance where it may be scattered, absorbed, or transmitted out of the bone. The photon was frequently moved until it either escapes from or was absorbed by the bone. If the photon escapes from the bone, the reflection or transmission of the photon was registered. If the photon was scattered, its scattering angle and its coordinate were registered. If the photon was absorbed, the position of the absorption 
was registered. This process is repeated until the desired number of photons (100) has been propagated. The coordinate of first interaction of photon and final scattering angle are the most important objects in radiotherapy by photon beams, hence they were determined by this simulation method [1-4].

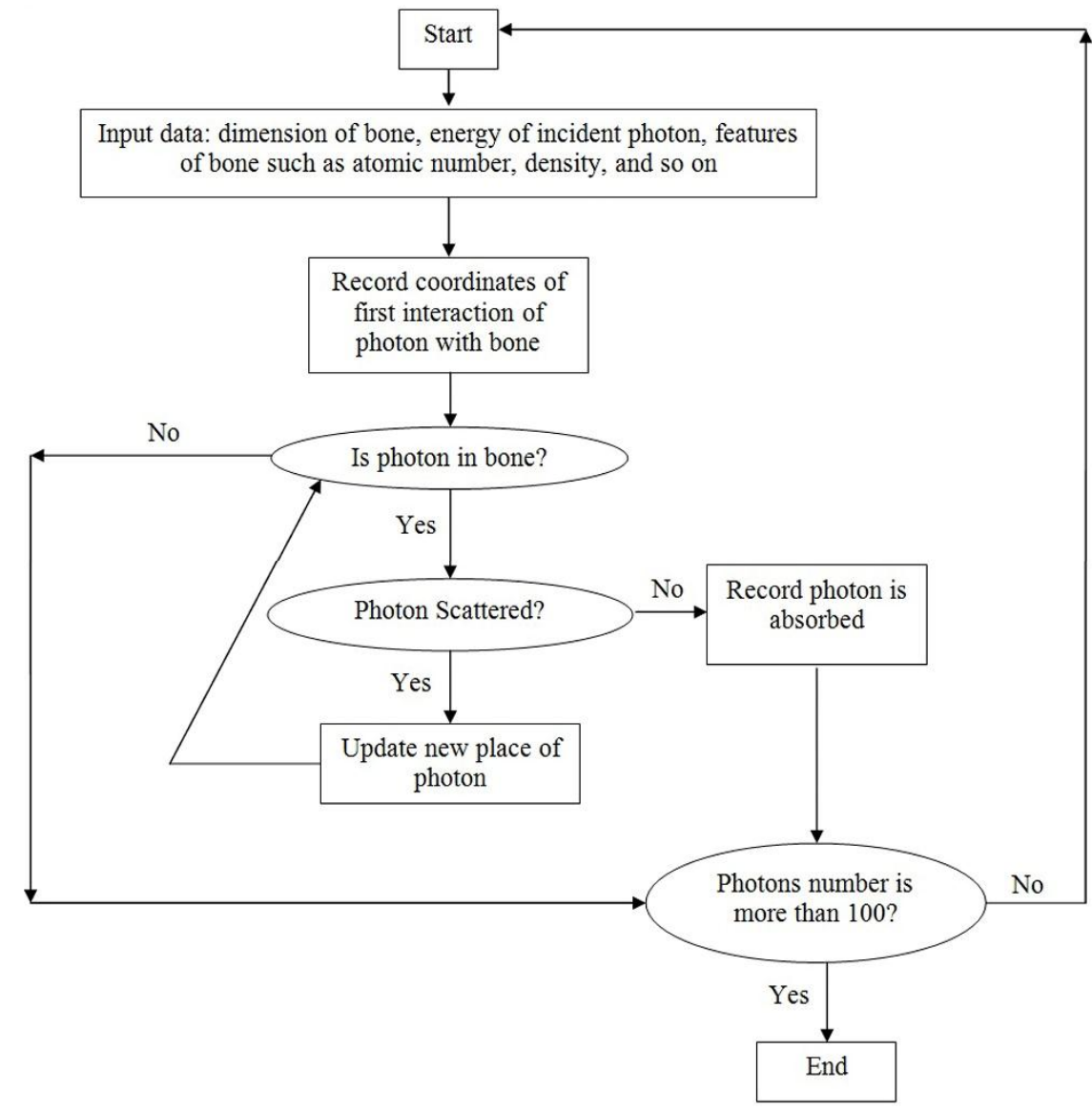

Figure 1. Flowchart of tracing of photons in bone by Monte Carlo Simulation

MCNP code

The emigration of photon beam in bone was simulated using MCNP-4C code. The simulation was carried out via the full continuous energy cross section accessible at the MCNP-4C library at $20^{\circ} \mathrm{C}$. The tally F5: $\mathrm{P}$ was used to obtain point flux of photons (particles/ $\mathrm{cm}^{2}$ ) at various depths in bone. Photons beam with 1, 5, and $10 \mathrm{MeV}$ energies as a photon source and point detector were used to determine the flux at each point in bone $\left(30 \times 30 \times 30 \mathrm{~cm}^{3}\right)[13]$.

\section{Result And Discussion}

The figures 2 and 3 illustrate simulation of photon propagation in bone using Monte Carlo simulation and MCNP code respectively. In these two figures, axis $\mathrm{Y}$ was different for various methods. Axis $\mathrm{Y}$ was number of photons, and point flux in Monte Carlo method, and MCNP code, respectively. These two different quantities were proportional to each other and their different were not important in present work, because the coordinate of first interaction of photon in bone and also penetration depth of photon in bone were in highest grade of significance. The axis $\mathrm{X}$ in these two figures indicates the penetration depth of photon in bone. As shown in figures 2 and 3 the depth of first interaction of photon in bone versus energies of 1,5 , and $10 \mathrm{MeV}$ were observed in $2.26 \mathrm{~cm}, 2.90 \mathrm{~cm}$, and $4.40 \mathrm{~cm}$ using the MC method, and $2 \mathrm{~cm}, 3 \mathrm{~cm}$, and $4 \mathrm{~cm}$ using MCNP code, respectively. The results show satisfactory consistent between MCNP code and MC simulation results. The deviation of these results may be due to this reason that in MC method, only two cross sections of photoelectric effect and Compton scattering were used and other cross sections were omitted because of their diminutive, although MCNP code uses all photon interactions as well electrons produced by the interactions of the photons in bone. 


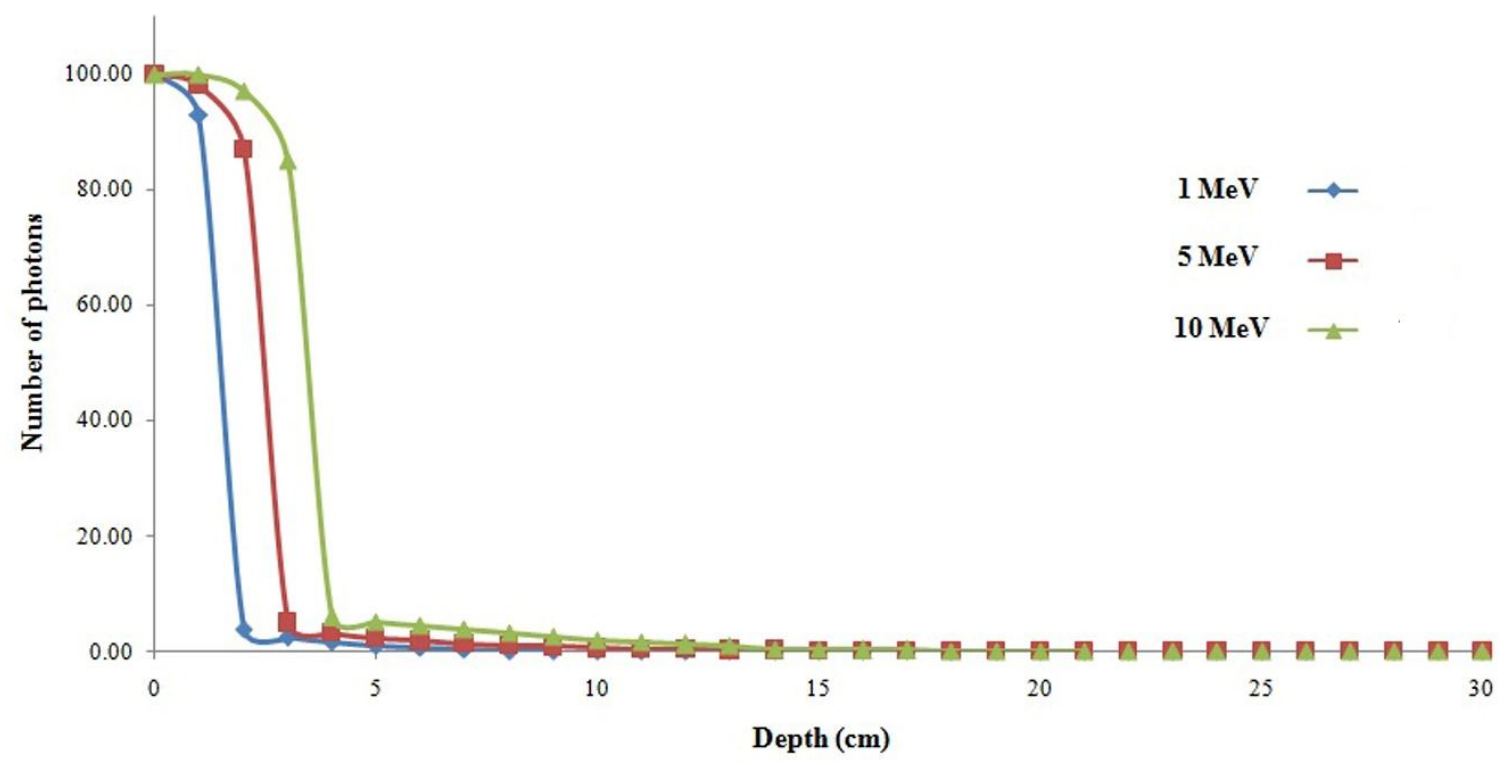

Figure 2. The number of photons with 1,5 , and $10 \mathrm{MeV}$ energies versus different depth of bone using Monte Carlo method

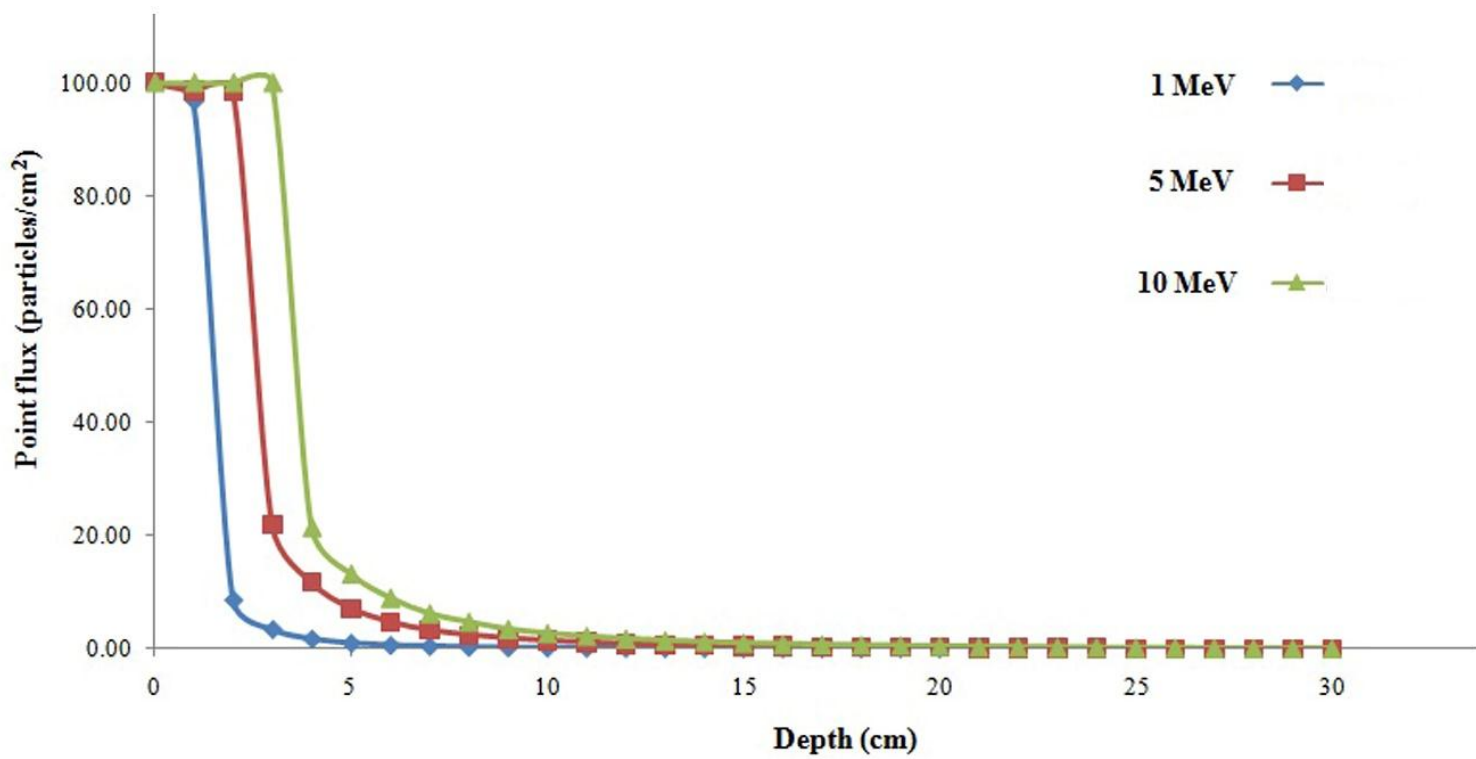

Figure 3. The point flux of photons with 1, 5, and $10 \mathrm{MeV}$ energies versus different depth of bone using MCNP code

Primary photon beam is widened during cross through in matter. The exiting angle of initial photon beam after pass through bone $\left(30 \times 30 \times 30 \mathrm{~cm}^{3}\right)$ was obtained $33.56,20.20$, and 12.44 degreesversus 1,5 , and $10 \mathrm{MeV}$ energies, respectively by MC method. Finding of the coordinate of first interaction of photon versusdifferent energies in matteras well as exiting angle of photon from matter is necessary in radiotherapy and it can decrease damage of photon beam on health tissue. Therefore, finding of present study may be useful for radiotherapy experts.

\section{Conclusion}

This study has presented a MC simulation for the transport of photon with different energies in bone. This feature enables the simulation of optical homographs with complex structures, such as small animal phantoms and will supply a way to better understand photon propagation issues and hence optimize reconstruction algorithms. The exiting angle of initial photon beam after pass through bonewas 33.56, 20.20, and 12.44 degrees versus 1,5 , and $10 \mathrm{MeV}$ energies, respectively by MC method. The result of MC method was 
evaluated by results of MCNP code. Satisfactory consistent between MCNP code and MC simulation results were observed. Finding of present study may be useful for radiotherapy experts. As a future work, simulation of photon propagation in multilayer material consists of tissue, muscle, bone can be investigated.

\section{Acknowledgments}

We thank the Prof. Dr. Ariai and Prof. Dr. Binesh (from Payam Noor University of Mashhad) for their assistance.

\section{References}

[1] Crowe, S. B. 2011. The development of Monte Carlo techniques for the verification of radiotherapy treatments. Ph.D. thesis. Queensland University of Technology, Australia.

[2] Moreno, M. Z. 2005. Monte Carlo simulations for dosimetric verification in photon and electron beam radiotherapy. Ph.D. thesis. Universidade Nova De Lisboa, Portugal.

[3] Spiga, J. 2010. Monte Carlo simulation of dose distributions for synchrotron microbeam radiation therapy. Ph.D. thesis. UniversitaDegliStudi Di Cagliari, Italy.

[4] Sidek, M. A. J. 2010. Monte Carlo investigations of radiotherapy beams: studies of conventional, stereotactic and unflattened beams. Ph.D. thesis. The University of Birmingham, United Kingdom.

[5] Verhaegen, F. \&Seuntjens, J. 2003. Monte Carlo modeling of external radiotherapy photon beams. Journal of Physics in Medicine and Biology. 48(21): R107.

[6] Sempau, J., Reyes, A. S., Salvat, F., Tahar, H. O., Jiang, S. B., \&Varea, J. M. F. 2001. Monte Carlo simulation of electron beams from an accelerator head using PENELOPE. Journal of Physics in Medicine and Biology. 46, 1163-1186.

[7] Wang, L., Jacques, S. L., \& Zheng, L. 1995. MCML-Monte Carlo modeling of light transport in multi-layered tissues. Computer Methods and Programs in Biomedicine, 47(2): 131-146.

[8] Hubbell, J.H. 2006. Review and history of photon cross section calculations. Phys. Med. Biol. 51, R245-R262.

[9] Johns, H.E. \& Cunningham, J.R. 1983. The Physics of Radiology, Fourth Edition. Charles C. Thomas, Springfield, Illinois

[10] Leo, R.W. 1987. Techniques for Nuclear and Particle physics Experiment: A how - to approach. Springer, Verlag.

[11] Tayal, D.C. 1982. Nuclear Physics. Himalaya Publishing House, Bombay.

[12] Katukuri, V. M., 2005. Photon transport in turbid media: simulation and experimental study. Ph. D. thesis. Sri Satya Sai University, India.

[13] Briesmeister, J.F. 1997. MCNP - a General Monte Carlo N-Particle Transport Code (Version C), Oak Ridge National Lab. 